\title{
Sarcopenia: Uma revisão narrativa das definições
}

\author{
Virgílio G. Moreira, ${ }^{1 *}$ Roberto A. Lourenço ${ }^{1,2}$
}

\section{Resumo}

Introdução: A sarcopenia é uma condição clínica associada ao envelhecimento. Está diretamente relacionada com desfechos indesejáveis à população idosa. Tem grande potencial terapêutico e apresenta elevada prevalência, de acordo com os critérios diagnósticos adotados. Objetivos: Realizar uma revisão sobre as principais definições de sarcopenia utilizadas em estudos epidemiológicos. Métodos: Trata-se de uma revisão narrativa com foco na busca de publicações sobre o tema utilizando palavras-chave por meio de linguagens boleanas via Medical Subheadings no PubMed: "sarcopenia", "diagnosis", "aged" e "epidemiology". Do total de 1058 citações, 14 foram selecionadas. Resultados: Os trabalhos analisados apresentam uma perspectiva histórica da sarcopenia englobando sua definição original - somente massa muscular - até os trabalhos subsequentes que agregaram força e, posteriormente, desempenho funcional aos seus critérios diagnósticos. Conclusões: Apesar dos trabalhos mais recentes identificarem a associação dos três critérios para definição de sarcopenia - massa, força muscular e desempenho funcional - são necessários outros estudos para verificar o melhor constructo para esta condição clínica, uma vez que o critério original ainda é utilizado nesta definição.

Descritores: Sarcopenia; Idosos; Diagnóstico.

\section{Abstract}

\section{Sarcopenia: a narrative review of definitions}

Introduction: Sarcopenia is a clinical condition associated with aging. It relates with undesirable outcomes in the elderly population, with great therapeutic potential, and presents high prevalence according to the diagnostic criteria adopted. Objectives: To present a narrative review on the main definitions of sarcopenia used in epidemiological studies. Methods: This study is based on the search of publications on the topic using keywords through Boolean languages via PubMed Medical Subheadings: "sarcopenia", "diagnosis", "aged" and "epidemiology". Of the total of 1058 citations, 14 were selected. Results: The papers present a historical perspective of sarcopenia from its original definition - only muscle mass - until the subsequent works that added strength, and after, functional performance to its diagnostic criteria. Conclusions: Although the most recent studies identify the association of the three criteria to define sarcopenia - mass, muscular strength and functional performance - further studies are needed to verify the best construct for this clinical condition since the original criterion is still used for its definition.

Keywords: Sarcopenia; Aged; Definition.
1. Laboratório de Pesquisa em Envelhecimento Humano - GeronLab. Universidade do Estado do Rio de Janeiro. Rio de Janeiro, RJ, Brasil.

2. Departamento de Medicina Interna. Hospital Universitário Pedro Ernesto. Universidade do Estado do Rio de Janeiro. Rio de Janeiro, RJ, Brasil.

\author{
*Endereço para correspondência: \\ Rua Engenheiro Enaldo Cravo Peixoto, 215/1113 \\ Rio de Janeiro, RJ. CEP: 20.540-106. \\ E-mail: virgilio.garcia.moreira@gmail.com \\ Revista HUPE, Rio de Janeiro, 2017;16(2):117-122 \\ doi: $10.12957 /$ rhupe.2017.37660 \\ Recebido em 17/07/2018. Aprovado em 03/08/2018.
}

\section{Resumen}

\section{Sarcopenia: una revisión narrativa de las defini- ciones}

Introducción: La sarcopenia es una condición clínica asociada al envejecimiento. Está directamente asociada a resultados indeseables a la población anciana y tiene gran potencial terapéutico. Esta condición presenta elevada prevalencia de acuerdo con los criterios diagnósticos adoptados. Objetivo: Realizar una revisión narrativa sobre las principales definiciones de sarcopenia utilizadas en estudios epidemiológicos. Metodología: Se trata de un estudio tipo revisión narrativa utilizando como base la búsqueda de publicaciones sobre el tema utilizando palabras clave a través de lenguajes boleanos vía Medical Subheadings, en PubMed: "sarcopenia", "diagnosis", "aged" y "epidemiología".Resultados/Discusión: Del total de 1058 citas, 14 fueron seleccionadas. Los trabajos analizados presentan una perspectiva histórica de la sarcopenia englobando su definición original - solamente masa muscular - hasta los trabajos subsiguientes que agregaron fuerza y posteriormente desempeño funcional a sus criterios diagnósticos. Conclusión: A pesar de los trabajos más recientes identificaren la asociación de los tres criterios para la definición de sarcopenia - masa, fuerza muscular y desempeño funcional - otros estudios son necesarios para verificar el mejor constructo para esta condición clínica ya que el criterio original todavía se utiliza para su condición definición.

Palabras clave: Sarcopenia; Ancianos; Diagnóstico. 


\section{Artigo de revisão}

\section{Introdução}

Os dados de pesquisas populacionais apresentam a modificação do perfil etário da população em todo o mundo. ${ }^{1}$ No Brasil, em menos de 70 anos, a expectativa de vida que era de 43 anos elevou-se para 74 anos de idade segundo dados do Instituto Brasileiro de Geografia e Estatística (IBGE). ${ }^{2} \mathrm{O}$ envelhecimento populacional é uma realidade consequente, entre outros fatores, à modificação do perfil sanitário, conquistas tecnológicas e conhecimento em saúde. ${ }^{3}$ Todavia, uma parcela desta população que envelhece é a que mais necessita de atenção e recursos. ${ }^{4} \mathrm{O}$ aumento da expectativa de vida traz consigo a observação de que quanto mais os anos avançam na vida de uma pessoa, maiores são as chances dela apresentar limitações funcionais e morbidades. ${ }^{5}$ Neste contexto, identificar tais condições são elementos centrais na abordagem do indivíduo em senescência.

A sarcopenia é uma das síndromes geriátricas que acometem esse subgrupo de indivíduos vulneráveis e, embora citada como problema de saúde desde a antiguidade, somente a partir do final dos anos 1980 passou a ser estudada. ${ }^{6,7}$ Descrita pela primeira vez por Rosenberg, apresenta-se diretamente associada a desfechos indesejáveis e alta morbidade. ${ }^{8-12} \mathrm{O}$ termo foi criado a partir de "sarx" (carne) e "penia" (perda ou redução), para apontar as alterações na composição corporal associadas ao envelhecimento, caracterizadas, principalmente, por redução da massa muscular esquelética. Rosenberg sugeriu que a gravidade desta condição estava associada ao risco potencial de redução da capacidade funcional. ${ }^{13} \mathrm{E}$, da mesma forma, constatou que nenhuma outra alteração associada ao envelhecimento era tão importante na determinação de desfechos indesejáveis quanto a redução na massa corporal magra. ${ }^{12,13}$

Ao longo das últimas quatro décadas o estudo sobre a sarcopenia foi crescente. Análises longitudinais apontaram que o pico de massa e força musculares ocorria entre a segunda e quarta décadas, declinando progressivamente com o avançar da idade. ${ }^{14} \mathrm{E}$, de forma mais acurada, representam uma perda de 0,6 a 1,29\% de massa muscular magra para homens e de 0,53 a 0,84\% de massa muscular magra para mulheres a partir dos 60 anos de idade. ${ }^{15-18}$ Além disso, do ponto de vista fisiopatológico, a sarcopenia foi identificada como multifatorial. Está conectada diretamente ao envelhecimento cronológico de sistemas orgânicos como neuromuscular, endócrino, além de ter relação com metabolismo proteico, inflamação e gasto energético. ${ }^{19-21}$

Apesar de todo conhecimento das últimas décadas sobre esta condição, uma importante questão encontra-se ainda aberta: como definir adequadamente a sarcopenia? ${ }^{14}$ Os trabalhos iniciais identificavam esta condição exclusivamente através do componente muscular.22 Posteriormente, foi acrescida à sua definição força e função musculares. ${ }^{23}$ Dada a necessidade de identificação desta condição e considerando seu potencial reabilitativo, o presente trabalho tem como objetivo realizar uma revisão narrativa sobre as definições de sarcopenia analisando os principais estudos sobre o tema.

\section{Metodologia}

Trata-se de uma revisão narrativa, englobando a literatura pertinente por meio de busca em um único repositório bibliográfico - PubMed-, utilizando linguagem MeSH com os unitermos "sarcopenia", "diagnosis", "aged", "epidemiology"utilizando as linguagens boleanas AND, OR cerceando as datas de busca até 2015 e artigos em língua inglesa.

\section{Resultados}

Foram observadas um total de 1058 citações envolvendo as palavras-chave. Após a leitura dos títulos dos artigos, 52 publicações mostraram-se relevantes para o presente estudo. Destas, após leitura dos resumos, 14 discutiam sobre os critérios diagnósticos com aplicação da sarcopenia (Tabela 1).

\section{Discussão}

Os achados dos trabalhos denotaram que os testes utilizados para mensurar a massa muscular na década de 1980 eram de alto custo, com grande exposição radioativa e pouco acessíveis. De fato, Rosemberg definiu o termo sarcopenia e toda a construção de sua definição deu-se a partir de então. ${ }^{13}$ Ao longo desta década, pesquisadores já discutiam formas de medir este componente corporal de forma mais prática e efetiva. ${ }^{36,37} \mathrm{Em} 1994$, pesquisadores se reuniram sob a coordenação do $\mathrm{Na}$ tional Institute on Aging, com o objetivo de discutir o significado e os mecanismos da sarcopenia. Apesar do evento não ter trazido respostas definitivas sobre o que é a sarcopenia, propôs uma agenda de investigação, e reconheceu a importância de nomear o fenômeno de perda de massa muscular, atendendo ao interesse da comunidade científica de despertar a atenção para o problema. ${ }^{12}$ Durante toda a década de 1990, os primeiros estudos epidemiológicos analisaram a massa muscular 
por meio de métodos não invasivos, descrevendo a prevalência de sarcopenia e alguns de seus desfechos indesejáveis, utilizando como critério diagnóstico a medida de massa muscular, realizada por meio de estimativa antropométrica, bioimpedância (BIA) e dual-energy X-ray absorptiometry (DEXA). ${ }^{38-42}$

No estudo denominado New Mexico Elderly Survey, conduzido de 1993 a 1995, Baumgartner e colaboradores $^{22}$ analisaram uma coorte de indivíduos usando o DEXA. Os autores categorizaram como casos aqueles que apresentavam massa muscular de dois desvios-padrão abaixo da média de uma população jovem. A prevalência de sarcopenia foi de 16,9\% e 24,1\%, para homens e mulheres latinos, e de 13,5\% e 23,1\%, para homens e mulheres caucasianos, respectivamente. A condição apresentou-se associada à baixa atividade física - independentemente de etnia, idade, morbidade,

Tabela 1. Instrumentos de rastreio cognitivo em idosos validados para a população brasileira

\begin{tabular}{|c|c|}
\hline Autor & Definição de sarcopenia \\
\hline Baumgartner [22] & Massa muscular \\
\hline Melton [24] & Massa muscular \\
\hline Delmonico $[18,25]$ & Massa muscular \\
\hline Janssen [26] & Massa muscular \\
\hline Visser [27] & Massa e força muscular \\
\hline Sowers [28] & Massa e força muscular \\
\hline Hairi [29] & Massa e força muscular \\
\hline Goodpaster [30] & Massa e força muscular \\
\hline Landi [31] & $\begin{array}{l}\text { Massa, força muscular e } \\
\text { desempenho funcional }\end{array}$ \\
\hline Van Kan [32] & $\begin{array}{l}\text { Massa, força muscular e } \\
\text { desempenho funcional }\end{array}$ \\
\hline Fielding [33] & $\begin{array}{l}\text { Massa, força muscular e } \\
\text { desempenho funcional }\end{array}$ \\
\hline Muscaritoli [34] & $\begin{array}{l}\text { Massa, força muscular e } \\
\text { desempenho funcional }\end{array}$ \\
\hline Cruz-Jentoft [23] & $\begin{array}{l}\text { Massa, força muscular e } \\
\text { desempenho funcional }\end{array}$ \\
\hline Chen [35] & $\begin{array}{l}\text { Massa, força muscular e } \\
\text { desempenho funcional }\end{array}$ \\
\hline
\end{tabular}

Legenda: MM: Massa muscular; FM: força de preensão palmar; DF: desempenho funcional. obesidade, renda ou hábitos de vida -, e estava presente em mais de $40 \%$ dos indivíduos acima de 80 anos.

Em 2000, Melton e colaboradores ${ }^{24}$ aplicaram o mesmo critério, anteriormente descrito, proposto por Baumgartner e colaboradores ${ }^{22}$ para mensuração da massa muscular. Sua amostra foi constituída por indivíduos de 20 a 80 anos de idade. Tanto para homens, quanto mulheres, observaram uma perda de massa muscular esquelética apendicular (MMEA) associada a idade cronológica. A prevalência de sarcopenia para aqueles com 65 anos ou mais variou de $6 \%$ a 14,6\%, e foi associada, em homens, com dificuldade de deambular [OR: 5,21 (1,03-8,96) IC: 95\%], e, em mulheres, com o aumento de fraturas osteoporóticas [OR: 5,21 (1,63-16,7) IC: $95 \%]$.

No estudo Health, Aging and Body Composition (HABC), Delmonico e colaboradores ${ }^{25}$ acompanharam durante cinco anos a massa muscular esquelética (DEXA) em uma população de 2.976 indivíduos acima de 70 anos de idade. O objetivo do estudo foi comparar dois critérios diagnósticos de sarcopenia para predição de declínio funcional. No primeiro critério, a massa magra apendicular foi calculada por meio da divisão da massa muscular apendicular (MMA) pela altura (Alt) do indivíduo elevada ao quadrado (MMA/Alt²); no segundo, pelo ajuste da MMA por altura e gordura corporal total, através de regressão logística. Os autores concluíram que o segundo critério foi melhor para predizer a incapacidade funcional, provavelmente por agregar gordura corporal à definição de sarcopenia. ${ }^{41,43}$

Em 2000, Janssen e colaboradores ${ }^{26,44}$ também mensuraram a massa muscular, porém por meio de dois métodos - a bioimpedância (BIA) e a ressonância nuclear magnética (RNM). Utilizando a RNM, determinaram a distribuição da massa muscular esquelética em 468 indivíduos com idades entre 18 e 88 anos. Apesar de não relatarem a prevalência de sarcopenia, observaram que a redução da massa muscular se iniciava aos 30 anos e que os homens apresentavam maior massa muscular. Todavia, alterações mais expressivas só estavam presentes a partir dos 50 anos de idade, e esta alteração foi encontrada especialmente em membros inferiores. ${ }^{44} \mathrm{Em} 2002$, utilizando a BIA, analisaram uma coorte de mais de dez mil indivíduos com 18 anos ou mais, acompanhados no Third National Health and Nutrition Examination Survey Study (NHANES III). O objetivo foi estabelecer a prevalência de sarcopenia em idosos, e testar sua associação com incapacidade funcional e limitações físicas. Dados normativos de massa muscular dos indivíduos jovens da coorte (18 a 39 anos) foram utilizados para definir 


\section{Artigo de revisão}

pontos de corte diagnósticos de sarcopenia. ${ }^{45}$ No total, 4504 indivíduos acima de 60 anos foram analisados e classificados em três estratos: normais - indivíduos com massa muscular acima de menos 1 desvio-padrão; classe I - indivíduos com massa muscular entre menos 1 e menos 2 desvios-padrão; classe II - indivíduos com massa muscular abaixo de menos 2 desvios-padrão. Na classe I, a prevalência de sarcopenia foi de 47 e 59\%, para homens e mulheres, respectivamente. Na classe II, foi de $6 \%$ e $9 \%$, respectivamente. Ainda, a prevalência aumentou com a idade e a redução de massa muscular estava diretamente associada ao comprometimento funcional e limitações físicas.

Até o final do século passado a massa muscular predominou nas definições de sarcopenia; no entanto, alguns estudos publicados neste mesmo período propunham que, além da massa, a força muscular também estaria associada a um pior desempenho funcional. ${ }^{46,47}$ Em 2000, Visser e colaboradores ${ }^{27}$ testaram esta hipótese em 449 indivíduos de 65 anos ou mais. Analisaram a associação entre desempenho funcional e massa e força musculares, usando DEXA e dinamômetro manual, respectivamente. Para verificação do desempenho funcional, os pesquisadores utilizaram o tempo dispendido para caminhar três metros e o teste de levantar e sentar em uma cadeira por cinco vezes. A baixa força muscular, mas não a massa muscular, foi associada à redução da funcionalidade em membros inferiores, para ambos os sexos.

Em 2005, Sowers e colaboradores ${ }^{28}$ aferiram a massa muscular pela BIA em 712 mulheres de 34 a 59 anos e avaliaram sua associação com força de membros inferiores em cadeira isométrica. Nos três anos de acompanhamento, houve redução de força muscular e 9\% perdeu mais de 2,5 kg de massa. Apesar desta coorte apresentar mulheres até 59 anos, a idade foi diretamente associada à redução de força. Em 2010, Hairi e colaboradores $^{29}$ também analisaram a associação de força e massa muscular com limitação funcional e desempenho físico em 1612 homens com 70 anos ou mais. Foram considerados anormais os indivíduos no percentil 20 para massa muscular pelo DEXA e força muscular pelo dinamômetro manual e de quadríceps. Assim como em Sowers e colaboradores, ${ }^{40}$ a força muscular foi, isoladamente, a variável que mostrou maior associação com limitações físicas e incapacidade.

Em 2006, Goodpaster e colaboradores ${ }^{30}$ mensuraram a massa muscular pelo DEXA e força de membros inferiores pelo extensor isocinético em 1880 indivíduos com 70 anos ou mais. Os objetivos deste estudo foram descrever a modificação de massa e força musculares ao longo de três anos e determinar se a modificação na massa muscular estava associada ao declínio da força muscular em idosos. Os autores concluíram que a redução de massa muscular estava associada à redução da força; porém, o declínio da força foi muito mais rápido do que o da massa muscular.

Como descrito acima, durante a década de 1990, a definição conceitual e operacional predominante de sarcopenia baseou-se sobretudo na massa muscular e suas formas de aferição. Na década seguinte, novas evidências incluíram a força muscular na definição de sarcopenia. A partir de 2008, informações relevantes sobre a associação de funcionalidade, desfechos negativos de saúde e sarcopenia, geraram várias propostas de modificação na definição da síndrome, agregando a variável desempenho funcional à massa e à força musculares. $^{32,48-50}$

Tendo em vista esta nova orientação diagnóstica, Landi e colaboradores ${ }^{31}$ utilizaram massa muscular pelo BIA, força pelo dinamômetro manual e desempenho funcional pela aferição da velocidade da marcha para identificar a prevalência de sarcopenia e fatores associados em indivíduos acima de 70 anos. A prevalência de sarcopenia foi de $32,8 \%$ associada ao sexo masculino [OR:13,3 (3,5-50,6) IC:95\%], doença cerebrovascular [OR: 5,1 (1,03-25,8) IC: 95\%] e osteoartrite [OR: 7,2 (225,9) IC: 95\%]. Aqueles com índice de massa corporal (IMC) acima de $21 \mathrm{~kg} / \mathrm{m}^{2}$ e atividades físicas de 1 hora ou mais ao dia apresentaram menor risco de sarcopenia. Na cidade do México, também avaliando massa muscular, força e desempenho em 345 indivíduos com 70 anos ou mais, Arango-Lopera e colaboradores ${ }^{51}$ encontraram uma prevalência de 33,6\% de sarcopenia.

Diante da multiplicidade de critérios e métodos de aferição propostos por pesquisadores em todo mundo, a partir de 2009, várias sociedades científicas criaram forças-tarefa com o objetivo específico de abordar uma série de questões não resolvidas sobre sarcopenia. ${ }^{23,32-35}$ Em 2009, Abellan Van Kan e colaboradores, ${ }^{32}$ reunidos na Carla Task Force on Sarcopenia, propuseram a massa e força musculares, além de desempenho funcional, como itens para o diagnóstico da sarcopenia.

Ainda em 2009, reunidos no International Working Group on Sarcopenia(IWGS), Fielding e colaboradores ${ }^{33}$ propuseram sarcopenia como perda de massa e função musculares associadas ao envelhecimento. Como em outras publicações, aquiesceram que a sarcopenia é multifatorial, composta por aspectos endócrinos, inflamatórios e deficiências nutricionais. Recomendaram a 
aferição da massa muscular por meio do DEXA - pontos de corte sugeridos por Baumgartner e colaboradres ${ }^{22}$-, e o desempenho funcional avaliado pela velocidade da marcha - ponto de corte de $1 \mathrm{~m} / \mathrm{s}^{33}$

Em 2010, no consenso intitulado Cachexia-Anorexia in Chronic Wasting Diseases and Nutrition in Geriatrics, Muscaritoli e colaboradores ${ }^{34}$ endossaram a proposta acima descrita de Fielding e colaboradores. ${ }^{33}$ Entretanto, sugeriram pontos de corte distintos: massa muscular de 2 desvios-padrão abaixo da média de adultos jovens ajustadas para sexo e etnia da população em estudo e velocidade da marcha de $0,8 \mathrm{~m} / \mathrm{s}$, independente de sexo, idade ou outras variáveis demográficas.

Também em 2010, a força tarefa reunida no European Work Group on Sarcopenia in Older People(EWGSOP) recomendou que alterações na massa, força e desempenho funcional estariam entre os critérios diagnósticos da sarcopenia. ${ }^{23}$ Foi proposta uma classificação em categorias e estágios. Quanto à categoria, primária (ou relacionada à idade), quando não existe uma causa evidente; ou secundária, quando uma ou mais causas são evidentes. Quanto ao estágio, para sarcopenia primária, em pré-sarcopenia, quando ocorre redução apenas na massa muscular; sarcopenia, que se caracteriza por redução na massa muscular e/ou, força muscular; e sarcopenia grave, quando há perda dos três itens: massa, força muscular e desempenho funcional (Tabela 2). Este trabalho foi um dos que gerou mais replicações nos últimos 7 anos. ${ }^{52-54}$

Em 2014, os mesmos critérios - massa muscular, força e desempenho funcional - também foram incluídos nos critérios diagnósticos de sarcopenia para o relatório do consenso do The Asian Working Group for Sarcopenia (AWGS). ${ }^{35}$ A sugestão dos métodos de aferição para cada um dos itens foi semelhante àqueles do EWGSOP. Contudo, com o objetivo de respeitar as características fenotípicas de sua população, propõem pontos de corte desenvolvidos em estudos com populações asiáticas.

Tabela 2. Sarcopenia segundo critérios do European Work Group on Sarcopenia in Older People (EWGSOP)

\begin{tabular}{ccccc}
\hline Estágio & $\begin{array}{c}\text { Massa } \\
\text { Muscular }\end{array}$ & $\begin{array}{c}\text { Força } \\
\text { Muscular }\end{array}$ & Desempenho \\
\cline { 2 - 4 } Pré-Sarcopenia & $\downarrow$ & & \\
Sarcopenia & $\downarrow$ & $\downarrow$ ou & $\downarrow$ \\
$\begin{array}{c}\text { Sarcopenia } \\
\text { Grave }\end{array}$ & $\downarrow$ & $\downarrow$ & $\downarrow$ \\
\hline
\end{tabular}

Fonte: Cruz-Jentoft et al. ${ }^{23}$.

\section{Conclusões}

O presente estudo observa que as definições de sarcopenia sofreram modificações ao longo dos últimos anos. Entretanto, dada a multiplicidade de critérios - até hoje a definição isolada de alguns dos itens é utilizada -, são necessários maiores estudos calcados em desfechos robustos para que seu constructo possa ser adequadamente estabelecido.

\section{Referências}

1. World Health Organization (WHO). World report on ageing and health. Geneva, Switzerland: World Health Organization; 2015.

2. Instituto Brasileiro de Geografia e Estatística (IBGE). Síntese de indicadores sociais: uma análise das condições de vida da população brasileirea. Rio de Janeiro: IBGE; 2015.

3. Canedo AC, Lopes CS, Lourenco RA. Prevalence of and factors associated with successful aging in Brazilian older adults: Frailty in Brazilian older people Study (FIBRA RJ). Geriatr Gerontol Int. 2018;18(8):1280-1285. doi: 10.1111/ ggi. 13334.

4. Moreira VG, Lourenco RA. Prevalence and factors associated with frailty in an older population from the city of Rio de Janeiro, Brazil: the FIBRA-RJ Study. Clinics (Sao Paulo). 2013;68(7):979-85

5. Cesari M, Marzetti E, Thiem U, Perez-Zepeda MU, et al. The geriatric management of frailty as paradigm of "The end of the disease era". Eur J Case Rep Intern Med. 2016;31:11-4.

6. Rizzoli R, Reginster JY, Arnal JF, et al. Quality of life in sarcopenia and frailty. Calcif Tissue Int. 2013;93(2):101-20.

7. Inouye SK, Studenski S, Tinetti ME, et al. Geriatric syndromes: clinical, research, and policy implications of a core geriatric concept. J Am Geriatr Soc. 2007;55(5):780-91.

8. $\mathrm{Wu} \mathrm{CH}$, Yang $\mathrm{KC}$, Chang $\mathrm{HH}$, et al. Sarcopenia is related to increased risk for low bone mineral density. J Clin Densitom. 2013;16(1):98-103.

9. Woo J, Leung J, Sham A, et al. Defining sarcopenia in terms of risk of physical limitations: a 5-year follow-up study of 3,153 chinese men and women. J Am Geriatr Soc. 2009;57(12):2224-31.

10. Weber J, Gillain S, Petermans J. [Sarcopenia: a physical marker of frailty]. Rev Med Liege. 2010;65(9):514-20.

11. Visser M. Obesity, sarcopenia and their functional consequences in old age. Proc Nutr Soc. 2011;70(1):114-8.

12. Rosenberg IH. Sarcopenia: origins and clinical relevance. J Nutr. 1997;127(5 Suppl):990S-1S.

13. Rosenberg IH. Sarcopenia: origins and clinical relevance. Clin Geriatr Med. 2011;27(3):337-9.

14. Cederholm TE, Bauer JM, Boirie Y, et al. Toward a definition of sarcopenia. Clin Geriatr Med. 2011;27(3):341-53.

15. Fantin F, Di Francesco V, Fontana G, et al. Longitudinal body composition changes in old men and women: interrelationships with worsening disability. J Gerontol A Biol Sci Med Sci. 2007;62(12):1375-81.

16. Koster A, Ding J, Stenholm S, et al. Does the amount of fat mass predict age-related loss of lean mass, muscle strength, and muscle quality in older adults? J Gerontol A Biol Sci Med Sci. 2011;66(8):888-95.

17. Frontera WR, Hughes VA, Fielding RA, et al. Aging of skeletal muscle: a 12-y longitudinal study. J Appl Physiol. 2000;88(4):1321-6.

18. Delmonico MJ, Harris TB, Visser M, et al. Longitudinal study of 
muscle strength, quality, and adipose tissue infiltration. Am J Clin Nutr. 2009;90(6):1579-85.

19. Curcio F, Ferro G, Basile C, et al. Biomarkers in sarcopenia: $A$ multifactorial approach. Exp Gerontol. 2016;85:1-8.

20. Bijlsma AY, Meskers CG, Westendorp RG, et al. Chronology of age-related disease definitions: osteoporosis and sarcopenia. Ageing Res Rev. 2012;11(2):320-4.

21. Dhillon RJ, Hasni S. Pathogenesis and Management of Sarcopenia. Clin Geriatr Med. 2017;33(1):17-26.

22. Baumgartner RN, Koehler KM, Gallagher D, et al. Epidemiology of sarcopenia among the elderly in New Mexico. Am J Epidemiol. 1998;147(8):755-63.

23. Cruz-Jentoft AJ, Baeyens JP, Bauer JM, et al. Sarcopenia: European consensus on definition and diagnosis: Report of the European Working Group on Sarcopenia in Older People. Age Ageing. 2010;39(4):412-23.

24. Melton LJ, 3rd, Khosla S, Crowson CS, et al. Epidemiology of sarcopenia. J Am Geriatr Soc. 2000;48(6):625-30.

25. Delmonico MJ, Harris TB, Lee JS, et al. Alternative definitions of sarcopenia, lower extremity performance, and functional impairment with aging in older men and women. J Am Geriatr Soc. 2007;55(5):769-74.

26. Janssen I, Heymsfield SB, Baumgartner RN, et al. Estimation of skeletal muscle mass by bioelectrical impedance analysis. J Appl Physiol (1985). 2000;89(2):465-71.

27. Visser M, Deeg DJ, Lips $P$, et al. Skeletal muscle mass and muscle strength in relation to lower-extremity performance in older men and women. J Am Geriatr Soc. 2000;48(4):381-6.

28. Sowers MR, Crutchfield M, Richards K, et al. Sarcopenia is related to physical functioning and leg strength in middle-aged women. J Gerontol A Biol Sci Med Sci. 2005;60(4):486-90.

29. Hairi NN, Cumming RG, Naganathan V, et al. Loss of muscle strength, mass (sarcopenia), and quality (specific force) and its relationship with functional limitation and physical disability: the Concord Health and Ageing in Men Project. J Am Geriatr Soc. 2010;58(11):2055-62.

30. Goodpaster BH, Park SW, Harris TB, et al. The loss of skeletal muscle strength, mass, and quality in older adults: the health, aging and body composition study. J Gerontol A Biol Sci Med Sci. 2006;61(10):1059-64.

31. Landi F, Liperoti R, Fusco D, et al. Prevalence and risk factors of sarcopenia among nursing home older residents. J Gerontol A Biol Sci Med Sci. 2012;67(1):48-55.

32. Abellan van Kan G, Andre E, Bischoff Ferrari HA, et al. Carla Task Force on Sarcopenia: propositions for clinical trials. J Nutr Health Aging. 2009;13(8):700-7.

33. Fielding RA, Vellas B, Evans WJ, et al. Sarcopenia: an undiagnosed condition in older adults. Current consensus definition: prevalence, etiology, and consequences. International working group on sarcopenia. J Am Med Dir Assoc. 2011;12(4):249-56.

34. Muscaritoli M, Anker SD, Argiles J, et al. Consensus definition of sarcopenia, cachexia and pre-cachexia: joint document elaborated by Special Interest Groups (SIG) "cachexia-anorexia in chronic wasting diseases" and "nutrition in geriatrics". Clin Nutr. 2010;29(2):154-9.

35. Chen LK, Liu LK, Woo J, et al. Sarcopenia in Asia: consensus report of the Asian Working Group for Sarcopenia. J Am Med Dir Assoc. 2014;15(2):95-101.

36. Young A, Stokes M, Crowe M. The size and strength of the quadriceps muscles of old and young men. Clin Physiol. 1985;5(2):145-54.
37. Heymsfield SB, McManus C, Smith J, et al. Anthropometric measurement of muscle mass: revised equations for calculating bone-free arm muscle area. Am J Clin Nutr. 1982;36(4):680-90.

38. Fuller NJ, Laskey MA, Elia M. Assessment of the composition of major body regions by dual-energy $\mathrm{X}$-ray absorptiometry (DEXA), with special reference to limb muscle mass. Clin Physiol. 1992;12(3):253-66.

39. Brodowicz GR, Mansfield RA, McClung MR, et al. Measurement of body composition in the elderly: dual energy x-ray absorptiometry, underwater weighing, bioelectrical impedance analysis, and anthropometry. Gerontology. 1994;40(6):332-9.

40. Visser M, van den Heuvel E, Deurenberg P. Prediction equations for the estimation of body composition in the elderly using anthropometric data. Br J Nutr. 1994;71(6):823-33.

41. Heymsfield SB, Gallagher D, Visser M, et al. Measurement of skeletal muscle: laboratory and epidemiological methods. J Gerontol A Biol Sci Med Sci. 1995;50 Spec No:23-9.

42. Heymsfield SB, Gallagher D, Grammes J, et al. Upper extremity skeletal muscle mass: potential of measurement with single frequency bioimpedance analysis. Appl Radiat Isot. 1998;49(56):473-4.

43. Guglielmi G, Ponti F, Agostini M, et al. The role of DXA in sarcopenia. Aging Clin Exp Res. 2016;28(6):1047-60.

44. Janssen I, Heymsfield SB, Wang ZM, et al. Skeletal muscle mass and distribution in 468 men and women aged 18-88 yr. J Appl Physiol (1985). 2000;89(1):81-8.

45. Janssen I, Heymsfield SB, Ross R. Low relative skeletal muscle mass (sarcopenia) in older persons is associated with functional impairment and physical disability. J Am Geriatr Soc. 2002;50(5):889-96.

46. Berkman LF, Seeman TE, Albert M, et al. High, usual and impaired functioning in community-dwelling older men and women: findings from the MacArthur Foundation Research Network on Successful Aging. J Clin Epidemiol. 1993;46(10):1129-40.

47. Fried LP, Ettinger WH, Lind B, et al. Physical disability in older adults: a physiological approach. Cardiovascular Health Study Research Group. J Clin Epidemiol. 1994;47(7):747-60.

48. Pahor M, Manini T, Cesari M. Sarcopenia: clinical evaluation, biological markers and other evaluation tools. J Nutr Health Aging. 2009;13(8):724-8.

49. Newman AB, Kupelian V, Visser M, et al. Sarcopenia: alternative definitions and associations with lower extremity function. J Am Geriatr Soc. 2003;51(11):1602-9.

50. Bautmans I, Van Puyvelde K, Mets T. Sarcopenia and functional decline: pathophysiology, prevention and therapy. Acta clinica Belgica. 2009;64(4):303-16.

51. Arango-Lopera VE, Arroyo P, Gutiérrez-Robledo LM, et al. Prevalence of sarcopenia in Mexico City. European geriatric medicine. 2012;3(3):157-60.

52. Bano G, Trevisan C, Carraro S, et al. Inflammation and sarcopenia: A systematic review and meta-analysis. Maturitas. 2017;96:10-5.

53. Diz JB, Leopoldino AA, Moreira BS, et al. Prevalence of sarcopenia in older Brazilians: A systematic review and metaanalysis. Geriatr Gerontol Int. 2017;17(1):5-16.

54. Cruz-Jentoft AJ, Landi F, Schneider SM, et al. Prevalence of and interventions for sarcopenia in ageing adults: a systematic review. Report of the International Sarcopenia Initiative (EWGSOP and IWGS). Age Ageing. 2014;43(6):748-59. 